\title{
Application of Doppler Global Velocimetry to Supersonic Flows
}

\author{
James F. Meyers \\ NASA Langley Research Center \\ Hampton, Virginia 23681
}

\begin{abstract}
The design and implementation of Doppler Global Velocimetry (DGV) for testing in the Langley Unitary Plan Wind Tunnel is presented. The discussion begins by outlining the characteristics of the tunnel and the test environment, with potential problem areas highlighted. Modifications to the optical system design to implement solutions for these problems are described. Since this tunnel entry was the first ever use of DGV in a supersonic wind tunnel, the test series was divided into three phases, each with its own goal. Phase I determined if condensation provided sufficient scattered light for DGV applications. Phase II studied particle lag by measuring the flow about an oblique shock above an inclined flat plate. Phase III investigated the supersonic vortical flow field above a 75 -degree delta wing at 24 -degrees angle of attack. Example results from these tests are presented.
\end{abstract}

\section{Introduction}

The development of aerodynamic flow measurement technology has been classically triggered by the need for information necessary to understand a particular flow phenomenon. In many cases the resulting instrumentation system was a modification of pre-existing technology. For example, the need to determine the magnitude of aerodynamic lift on configurations in wind tunnels resulted in the adaptation of butcher's scales to measure the force applied. Likewise, weather manometers were adapted to make surface pressure measurements from static taps on model surfaces. Additional applications of the manometer, total pressure probes and Pitot-static tubes, addressed the need to determine the off-body flow field. Later, more advanced probes based on heat transfer technology, hot wires and hot films, were developed to investigate flow turbulence.

While these probes yielded a great deal of information about the fluid mechanics involved in aerodynamic research, their intrusive nature adversely affected the flow. In response to the need for nonintrusive measurements, Yeh and Cumins ${ }^{1}$ used newly developed laser technology along with an adaptation of radar technology to invent the laser Doppler 
velocimeter in 1964. Although this reference-beam laser Doppler velocimeter proved too complicated to be a practical instrument for flow diagnostics, it triggered the development of the fringe-type laser velocimeter and the laser transit anemometer. These two instruments have provided great insight in to wind tunnel and compressor flow field investigations, respectively.

Instrumentation technologies were not the only areas in which advances were being made. The aerodynamic community discovered that vortical flows generated a great deal of lift at high angles of attack. Complicated aircraft designs with many appendages, such as leading edge extensions, to produce vortical flows resulted in increased performance. The flow fields created by these designs proved to be far more complicated than from earlier configurations. Sequential measurements obtained with probes and laser velocimetry required a great deal of wind tunnel usage to yield measurement grid densities sufficient to provide the necessary detailed descriptions of these flows, especially as validation databases for the newly developing science of Computational Fluid Dynamics. The cost and declining availability of wind tunnel testing created the need for global measurement technology. Instrumentation developers replied with Particle Image Velocimetry for detailed studies of low speed flows, and Doppler Global Velocimetry ${ }^{2}$ for large mappings of higher speed flows.

The following is a description of the implementation of Doppler Global Velocimetry (DGV) in the Langley Unitary Plan Wind Tunnel. The primary purpose of this supersonic flow field investigation was to determine the ability of the technology to measure high-speed flows. Anticipated difficulties were the installation of the receiver optical systems at the selected viewing angles, particle dynamics, and the determination of velocity based on the expected large Doppler frequency shifts.

\section{The Unitary Plan Wind Tunnel}

The Langley Unitary Plan Wind Tunnel (UPWT) is a closed-circuit, continuous flow, variable density, compressor driven supersonic wind tunnel complex with two $1.2-\mathrm{x} 1.2$-meter test sections ${ }^{3}$. One test section is on a low speed leg, which has a Mach number range of 1.5 to 2.9. The other test section is on a high speed leg, with Mach numbers ranging from 2.3 to 4.6. The tunnel has sliding-block-type nozzles that allow continuous variation in Mach number while the facility is in operation. The maximum Reynolds number per foot varies from $6 \times 10^{6}$ to $11 \times 10^{6}$, depending on Mach number. The DGV flow field investigations were conducted in the high-speed test section, figure 1 . 


\section{Installation and Testing Constraints The Good News}

Application of Doppler Global Velocimetry needed good optical access, ample space about the test section to install the optical components, and flow seeding to obtain sufficient scattered light for good measurement quality. The UPWT had excellent optical access with nine 0.14-x 1.22meter schlieren quality, $3.8-\mathrm{cm}$ thick windows separated by $3.18-\mathrm{cm}$ thick support ribs on each side of the test section, figure 2. These windows were mounted in 1.76 -meter wide doors that served as the side walls of the test section. The doors required a 2 -meter clearance to fully open. Thus, these large open areas provided sufficient space next to the test section to install the optical system.

Operating the wind tunnel at dew points between $-6.7^{\circ} \mathrm{C}$ to $-1.1^{\circ} \mathrm{C}$ produced sufficient water condensation to visualize the flow field using light sheet techniques. These vapor screens have routinely been used to provide insight into flow phenomena about wind tunnel models under investigation ${ }^{5}$. Since DGV measurements only required sufficient scattered light to be detected by Charge-Coupled-Detector (CCD) video cameras ${ }^{4}$, it was anticipated that scattering laser light from the condensation would produce acceptable results.

Operationally, other characteristics of the UPWT were favorable for DGV measurements:

-Sufficient electrical service and cooling water was available for operation of an Argon ion laser.

-The test section was located in a large windowless room that had only two doors. Thus the test area was controllable for purposes of laser safety, and could be darkened to reduce background light levels.

- Since the model support structure was not only capable of changing angle of attack, but of translating 0.92 meters in the streamwise direction, it was not necessary to move the laser light sheet or the receiving optical system during the flow field investigation.

- The tunnel was capable of continuous operation which provided the opportunity to check the DGV measurements while the tunnel was running. 
Installation and Testing Constraints

The Bad News

Again, application of Doppler Global Velocimetry needed good optical access, ample space about the test section to install the optical components, and flow seeding to obtain sufficient scattered light for good measurement quality. Unfortunately, the characteristics outlined above only partially describe the installation and test constraints. While the optical access was excellent, the vertical support structures, figure 2, extended $20.3 \mathrm{~cm}$ from the window surface, figure 3 . These barriers prohibited direct viewing of the laser light sheet by the receiver optical systems at angles other than orthogonal to the windows.

Although sufficient room was available on both sides of the test section to locate the optics in any desired position, the need to enter the test section to perform DGV optical calibrations, and adjust and measure the model position limited optical placement to on e side of the test section. Further, no optical access was available from the top of the test section.

An attempt to use condensation as the seeding material was made during a previous Laser Transit Anemometer (LTA) investigation of the boundary layer above an inclined conical section. Unfortunately, the LTA was unable to obtain velocity measurements from the condensation particles although the scattered light levels were sufficient for normal operation. The inability to obtain measurements was either due to the particles being too small, less than 0.2 micron in diameter, or the particle number density so high that particles were constantly in both focused laser beams. Since condensation produced sufficient scattered light for vapor screen flow visualization, the LTA measurements most likely failed because of high particle number density. Thus, even though the vapor screen experience indicated that sufficient scattered light was available for DGV, the condensation particle size remained unknown along with the particle response to flow field characteristics.

Operationally, other characteristics of the UPWT were unfavorable for DGV measurements:

- The center of rotation for model angle of attack adjustment was located downstream of the test section, figure 3 .

- The model sting was not capable of vertical motion.

- Run time was split between the two test sections with one hour required to shift the air flow between the sections. 
-The high velocity produced Doppler shift frequencies greater than the frequency spanned along a single side of the Iodine absorption line, figure 4.

- The UPWT could only be operated on third shift (11:00 p.m. to 8:00 a.m.) because of electrical power constraints.

\section{Plan of Attack}

The test program was designed to consist of three phases of increasing complexity. The first phase would determine if the condensation would yield velocity images. The second would determine the size of the condensation particles by measuring the vertical velocity component imposed on the particles as they passed through an oblique shock. The third phase would investigate the supersonic vortical flow above a 75-degree delta wing at Mach 2.96 and Mach 4.6.

The DGV optical system consisted of an Argon ion laser, two two-component galvanometer scanner systems, and a single receiver optical system. Multiple velocity component measurements were obtained by using combinations of two laser light sheet propagation directions and two receiver positions. Since the optics could only be placed on one side of the facility, the second light sheet direction was obtained by passing the laser beam through the test section to a scanner system located on the far side of the tunnel. The scanner was mounted on a rail attached to the tunnel window vertical supports, allowing the test section door to be opened and closed without optical misalignment. The scanners were electronically controlled to produce light sheets of a desired fan angle. Additionally the light sheet could be rotated from the horizontal to the vertical planes. The galvanometer mirrors were oscillated at $130 \mathrm{~Hz}$ to sweep the laser beam through the measurement plane four times during the video camera field acquisition time of $1 / 60^{\text {th }}$ second.

During the first two test phases, the optical system was arranged with the receiver system orthogonal to the tunnel window to view an inclined light sheet in forward scatter. This arrangement kept the optics simple while maximizing the collected Mie scattered light. The laser beam was passed through the test section to the scanner on the far side of the tunnel. The scanner was set to create a light sheet fanned horizontally in the streamwise direction with an inclination angle of 33.5 degrees above the horizontal, figure 5 . This configuration measured the velocity component 16.75 degrees from vertical in the cross flow direction. Assuming the cross flow velocity was negligible, the measured velocity was expected to be zero for the empty tunnel test in 
Phase I. This component was also the most sensitive component to indicate the ability of the condensation particles to follow the flow behind the oblique shock in Phase II.

In Phase III, the optical components were rearranged to view a measurement plane aligned in the cross-tunnel direction orientated perpendicular to the delta wing, figure 6 . The two scan ners were aligned to place the opposing light sheets in the measurement plane. Since the light sheets could not be viewed directly because of the vertical support structures, a mirror was mounted to the support structure on the far side and aligned to provide a view of the light sheet from the optics side of the tunnel, figure 7 . The receiver optics system and reflecting mirror were set to the top of the opposing windows, respectively, to obtain the first view, then moved near the bottom of the windows to obtain the second view aligned along the delta wing surface. The combination of the two light sheets and the two receiver optical positions resulted in the ability to measure four velocity components.

\section{Will It Work?}

With the optical configuration designed and the test plan defined, the DGV system was constructed and installed in the UPWT, figures 8 and 9. A jig was built to hold the alignment target containing evenly spaced $\operatorname{dots}^{4}$ at the Phase I design inclination angle of 33.5 degrees, figure 10 . The jig was placed in the wind tunnel with the target plane aligned along the streamwise direction. The scanner system was adjusted to generate a laser light sheet that matched the target plane, figure 5. The receiver optical system was installed on the DGV support structure and aligned perpendicular to the tunnel window while viewing the alignment target, figure 9. A video analog divider ${ }^{4}$ was used to determine when the signal and reference video cameras were aligned. The divider output was monitored while the cameras were adjusted to minimize the mismatch in the dots, figure 11.

Following optical system align ment, the jig was removed and the tunnel prepared for testing. A sharp-edged flat plate was installed on the model support structure, inclined to -15 degrees, and positioned downstream of the measurement plane. With the plate located downstream, its presence would not influence the flow passing through measurement plane. The tunnel was started and set for a free stream Mach number of 2.5. Water was then injected into the tunnel settling chamber raising the flow humidity to the level needed to create condensation. Although the condensation was not visible in normal room lights, the scattered laser light was sufficient to produce good quality signal and reference images. The resulting signal and reference camera images were 
simultaneously acquired by a NASA-constructed dual frame grabber ${ }^{5}$ and passed to the data acquisition computer for storage and processing. The processed empty tunnel data is illustrated by the velocity image shown in figure 12. An X-Y plot of velocity measurements along a row of pixels shows that the velocity field is not uniform, figure 13. This was caused by the change in the measured velocity vector as the scanner swept the laser beam upstream / downstream. After calculating and removing the freestream contribution $\left(U_{\infty} \sin \alpha\right)$ in the velocity measurement, the vertical component was isolated, figures 14 and 15 . From these measurements it was concluded that water condensation provided sufficient scattered light for DGV measurements. Further, the measured velocities matched the expected characteristics for a supersonic flow through an empty tunnel.

The successful measurement of the empty tunnel flow field completed the Phase I portion of the test series. The Phase II portion was begun by moving the inclined flat plate into the measurement plane. The resulting measurement of the velocity field about the oblique shock above the plate is shown in figure 16. The freestream contribution was not removed to maintain the velocity profile across the shock. The particle lag was determined by viewing the velocity profile along a row of pixels as it crossed the shock. The row presented in figure 17 crossed the shock where the freestream contribution was negligible. While the velocity lag was only $2 \mathrm{~mm}$, it was greater than expected for the small condensation particles. One possible explanation was a characteristic of imaging optics - Modulation Transfer Function (MTF).

The MTF or sharpness factor is a measure of spatial frequency limit of the imaging optics and CCD camera. For example, a photograph taken with an f16 lens opening produces a sharper image than the same photograph taken with an f2 lens opening. The MTF of a CCD video camera is related to electronic charge leakage between adjacent pixels. The combined lens/camera MTF is measured by imaging a spatial step change in light intensity, and determining the kernel needed to match the measured response. The MTF for the present camera system is shown in figure 17 and found to approximate the measured velocity data. The theoretical velocity profiles, after convolution with the MTF, for 0.5 - and 1.0-micron particles are also shown in figure 17. From these measurements it was found that the apparent particle lag behind the shock was in fact due to the MTF of the camera system. Further, a comparison of the measured velocity profile with the theoretically expected lag for small particles indicated a reasonable match. Therefore, measurements of the supersonic velocity flow field could be made with DGV using scattered light from water condensation. 
An assessment of the first two phases was encouraging. The results from Phase I indicated that scattered light from condensation was sufficient to yield DGV measurements. The Phase II results indicated that a small vertical velocity could be detected within a high-speed streamwise flow. Further, the small velocity lag indicated that the condensation particles were very small. The successful completion of Phase II provided sufficient confidence to continue with the measurement of the complex flow in Phase III.

\section{The Goal Is Reached}

With the successful completion of the first two phases, the flat plate was replaced with a 75-degree delta wing, and the DGV optical system was reconfigured for the first of four desired measurement components. The image warping calibration was performed with the alignment target intersecting the 95-percent chord location and aligned orthogonal to the delta wing, figures 7 and 18. After both light sheets were aligned with the target, and the target images acquired for later dewarping calculations, the target was removed and the tunnel started with the Mach number set to 2.8. Step changes in condensation number density, caused by local flow conditions, delineating the main flow structures were seen using vapor screen visualization, figure 19 . The challenge in this phase was whether DGV could define this complicated flow structure. Further, could DGV even measure the large Doppler frequency shifts generated by this flow?

After the tunnel was started, the signal and reference images were acquired and examined to determine where the laser frequency should be tuned to obtain the best measurements. Two components $(-0.30 \mathrm{i}$ $-0.95 j+0.12 \mathrm{k}$ and $-0.25 \mathrm{i}-0.91 \mathrm{j}+0.33 \mathrm{k}$ ) yielded Doppler frequency shifts less than $500 \mathrm{MHz}$. Thus the laser could be tuned to a frequency near the top of the absorption curve, figure 4, while leaving sufficient bandwidth for the Doppler shifted frequency to be measured near the bottom. The other two components $(-0.90 \mathrm{i}+0.31 \mathrm{j}+0.32 \mathrm{k}$ and $-0.57 \mathrm{i}$ $+0.40 \mathrm{j}+0.72 \mathrm{k}$ ) yielded Doppler frequency shifts exceeding $1.0 \mathrm{GHz}$. This shift was greater than the side bandwidth of the absorption line. By tuning the laser to a frequency near the midpoint on the left side of the absorption curve, figure 4, the shift frequency would span the absorption well. Using the calibration of the entire absorption line, the Doppler shifts were then determined with the laser frequency on the left side and the scattered light frequency on the right.

The ability of DGV to measure these large Doppler frequency shifts and delineate the vortical flow structures is illustrated by the results shown in figures $20-23$. These results were obtained at the 95 -percent chord 
location with the delta wing set to 24 degrees angle of attack. The location and characteristics of the velocity flow structure were clearly seen, indicating that DGV was capable of providing global measurements of supersonic flows. Additionally, the high spatial resolutions obtained with DGV yielded measurement grid densities exceeding those typically used in Computational Fluid Dynamic simulations. Thus, velocity databases generated by DGV measurements could provide detailed verification of simulation codes.

\section{Summary}

The sequence of events necessary to conduct a flow field investigation using Doppler Global Velocimetry in a supersonic wind tunnel was presented. The sequence included characterization of the physical wind tunnel, flow field conditions, and required DGV system constraints. The step-by-step procedures used to reach the desired investigation of the vortical flow above a 75-degree delta wing were outlined. Preliminary measurements of the empty tunnel and oblique shock region above an inclined flat plate were presented, along with example velocity images from four velocity measurement components of the vortical flow above the delta wing. The results indicate that DGV was capable of producing multi-component velocity measurements of supersonic flows with grid densities sufficient for verification of Computational Fluid Dynamic simulations.

\section{References}

1. Yeh, Y.; and Cummins, H. Z.: Localized Fluid Flow Measurements with a He-Ne Laser Spectrometer. Applied Physics Letters, vol. 4, no. 10, pp. 176-178, May 1964.

2. Komine, H.: System for Measuring Velocity Field of Fluid Flow Utilizing a Laser-Doppler Spectral Image Converter. United States Patent 4,919,536, April 24, 1990.

3. Jackson, C. M., Jr.; Corlett, W. A.; and Monta, W. J.: Description and Calibration of the Langley Unitary Plan Wind Tunnel, NASA TP 1905, November 1981.

4. Meyers, J. F.; and Komine, H.: Doppler Global Velocimetry - A New Way to Look at Velocity. Laser Anemometry: Advances and Applications, 1991, eds. A. Dybbs \& B. Ghorashi, ASME, 1991. 
5. Meyers, J. F.: Doppler Global Velocimetry - The Next Generation? AIAA 17th Aerospace Ground Testing Conference, Paper 92-3897, Nashville, TN, July 6-8, 1992.

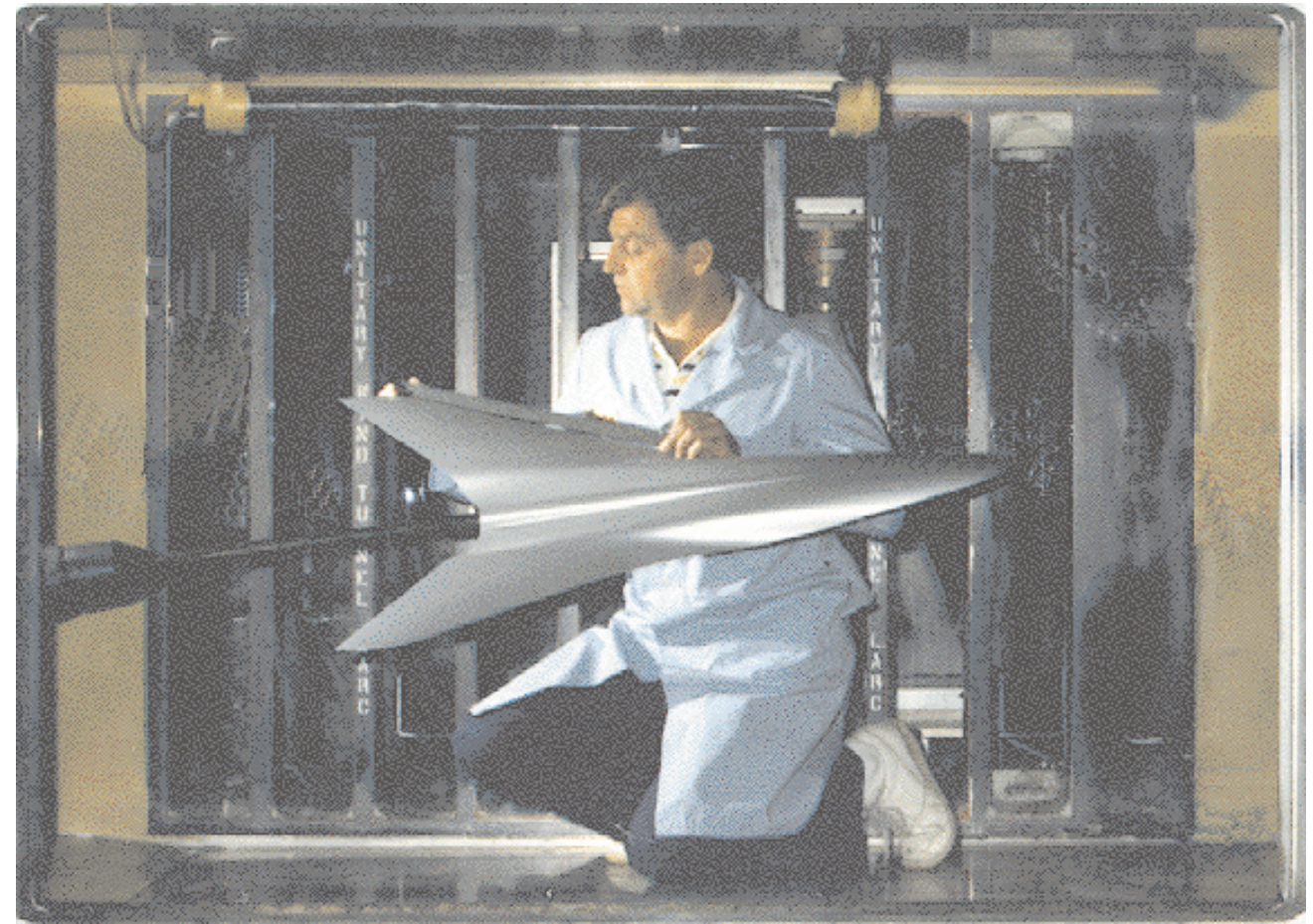

Figure 1.- Unitary Plan Wind Tunnel test section.

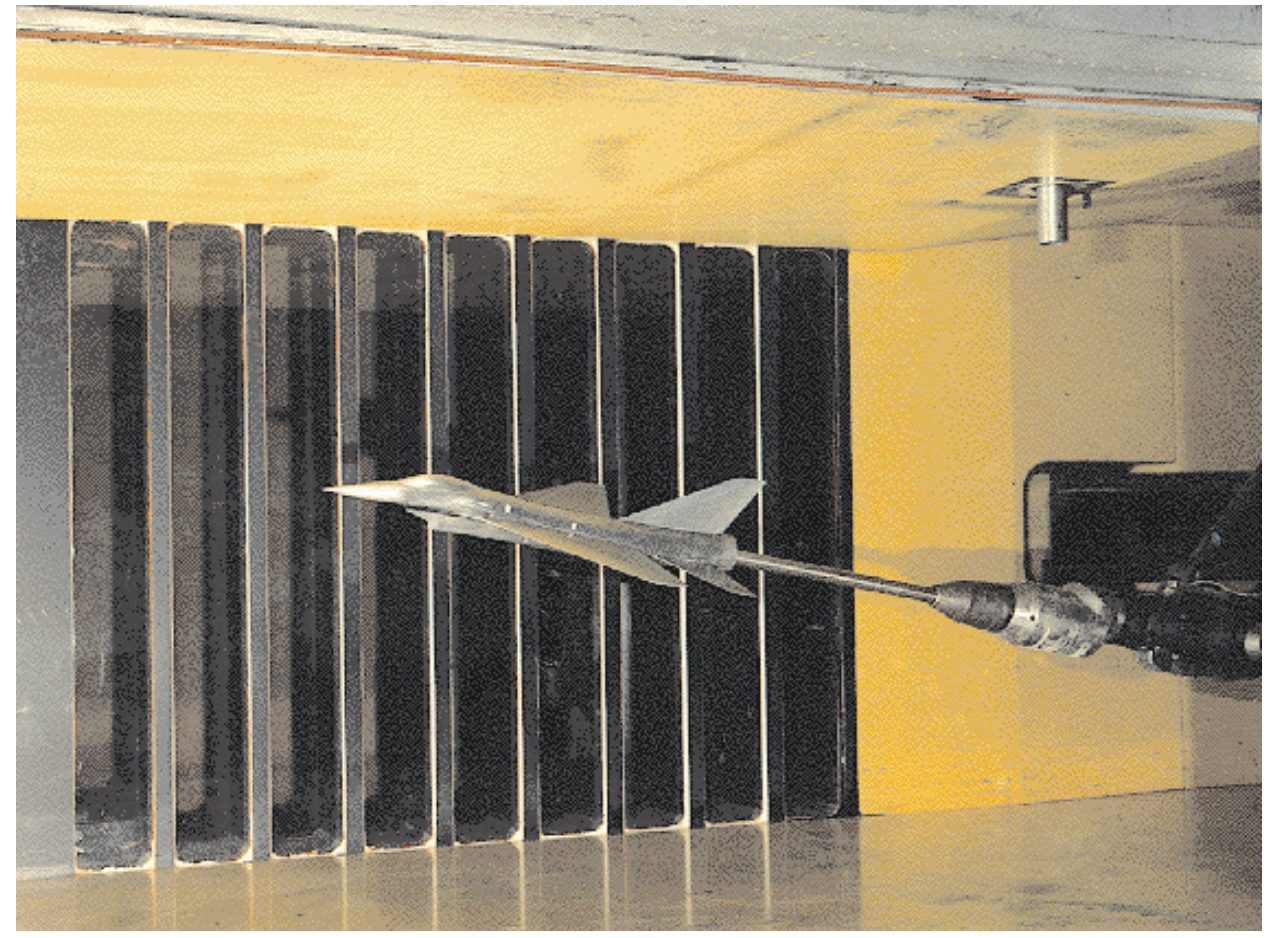

Figure 2.- Test section showing the windows and vertical support structure. 

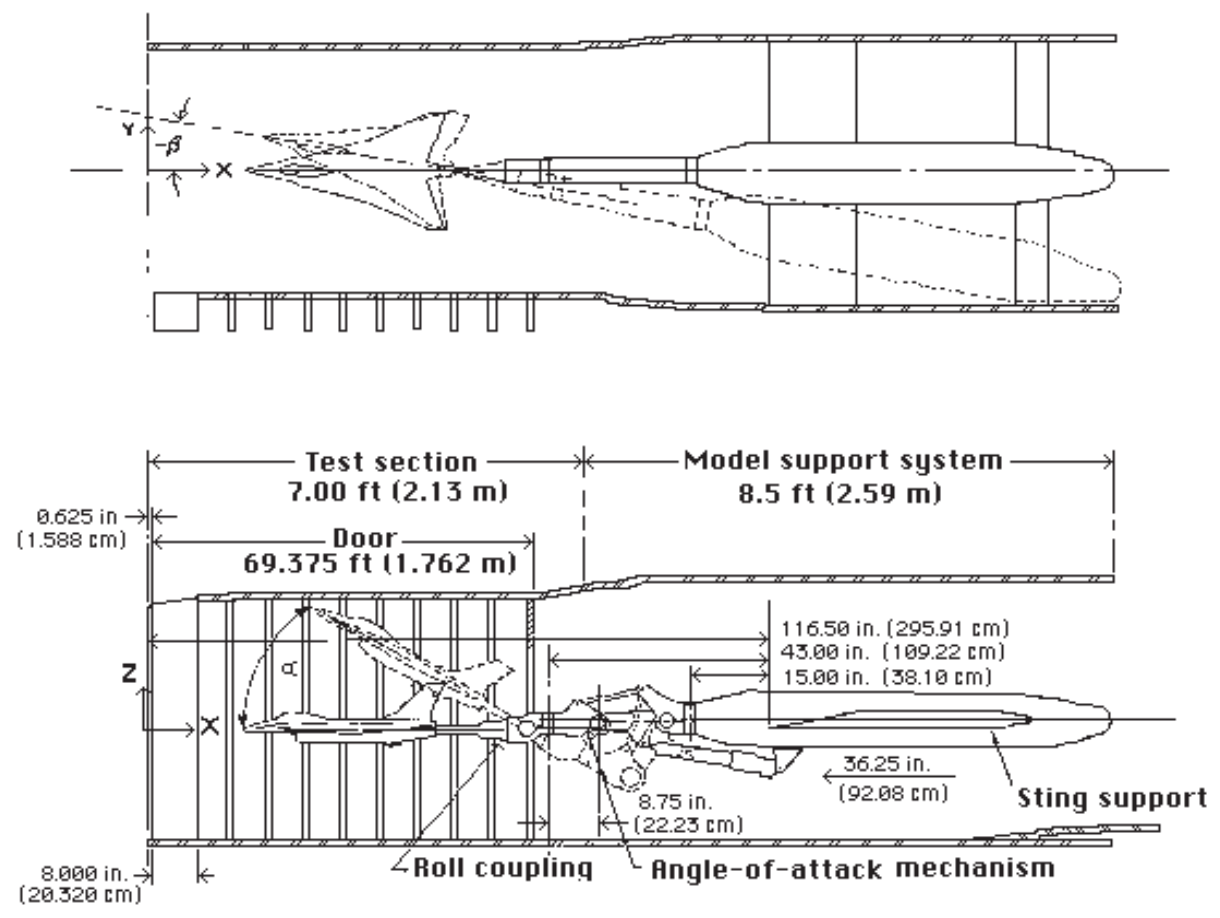

Test section and model support system

Figure 3.-Test section components and layout.

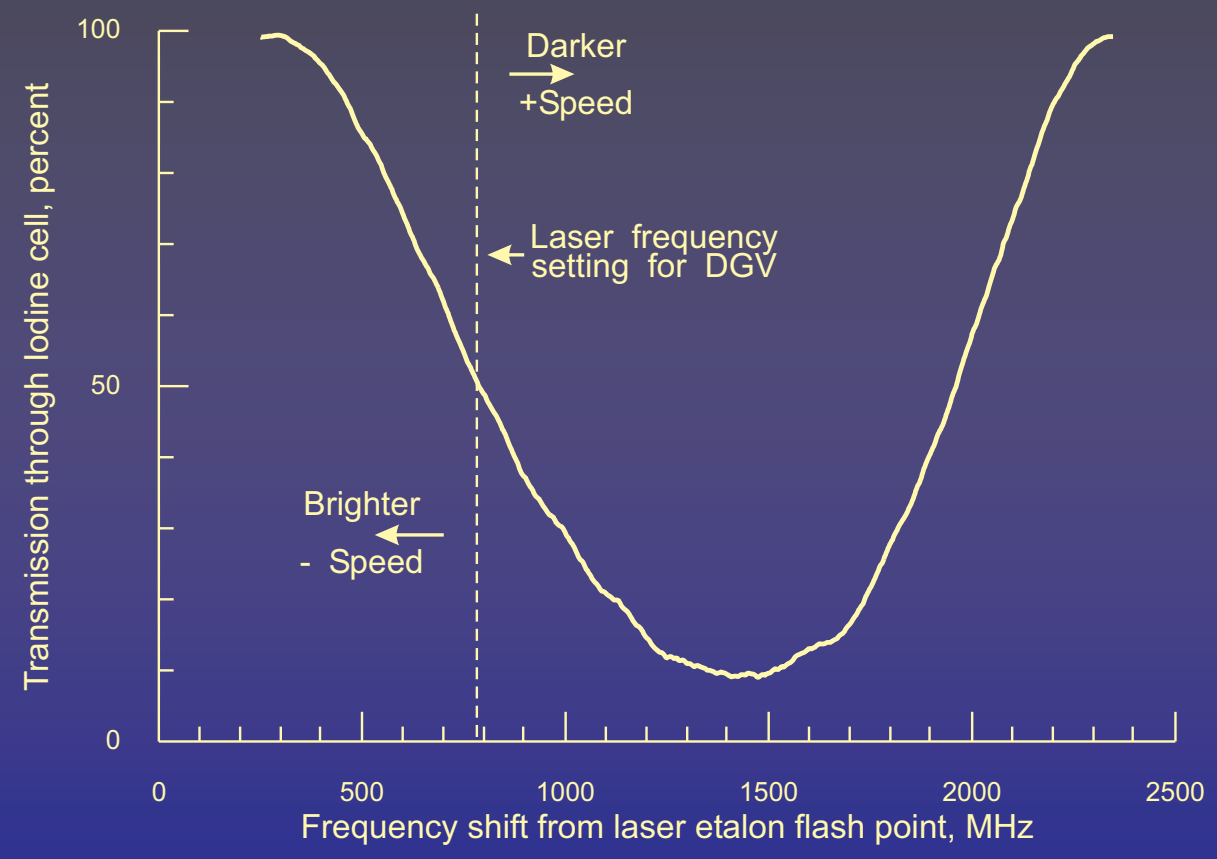

Figure 4.-Transfer function of the Iodine vapor cell. 


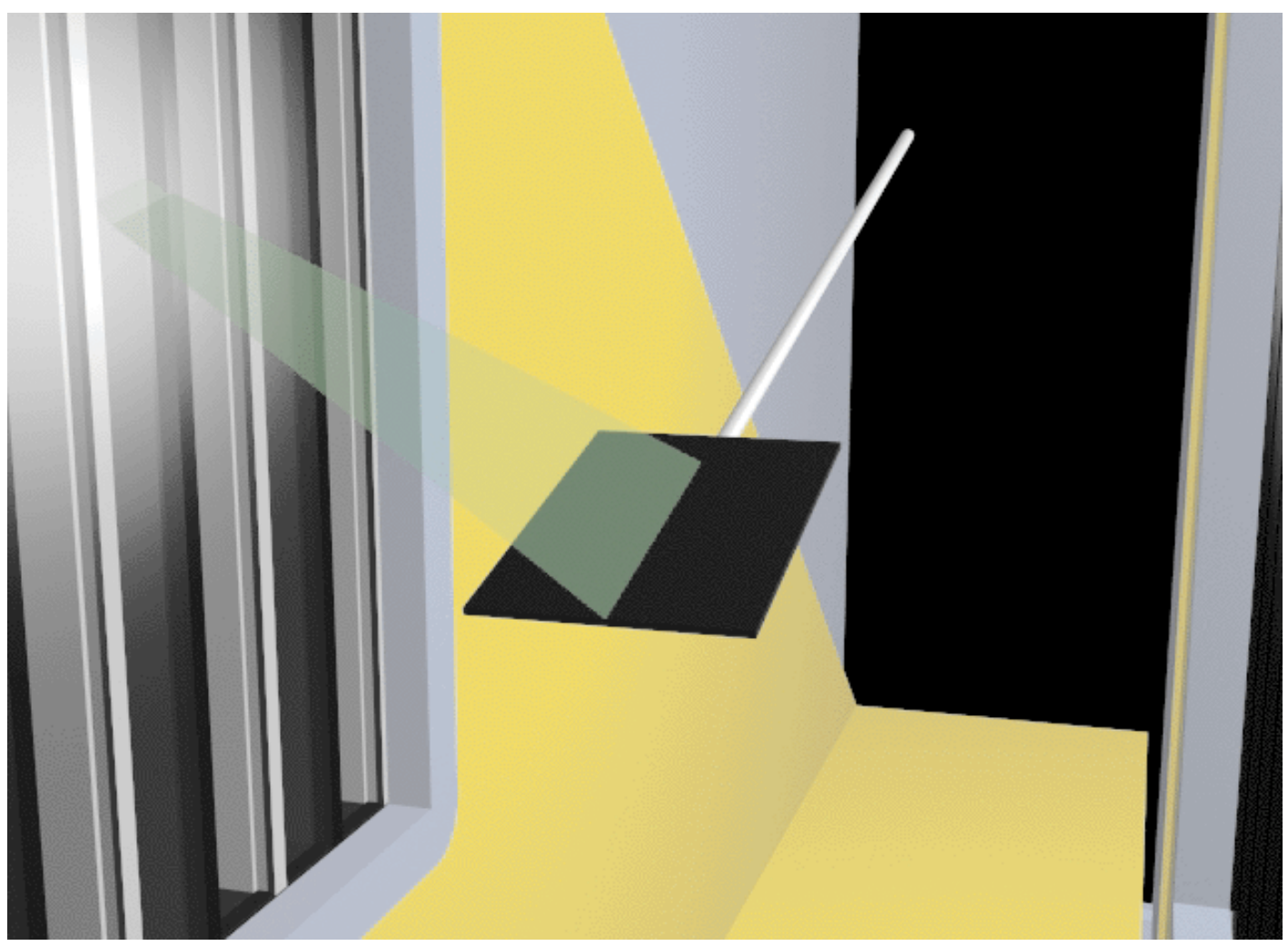

Figure 5.-Streamwise laser light sheet intersecting thesharp edged flat plate.

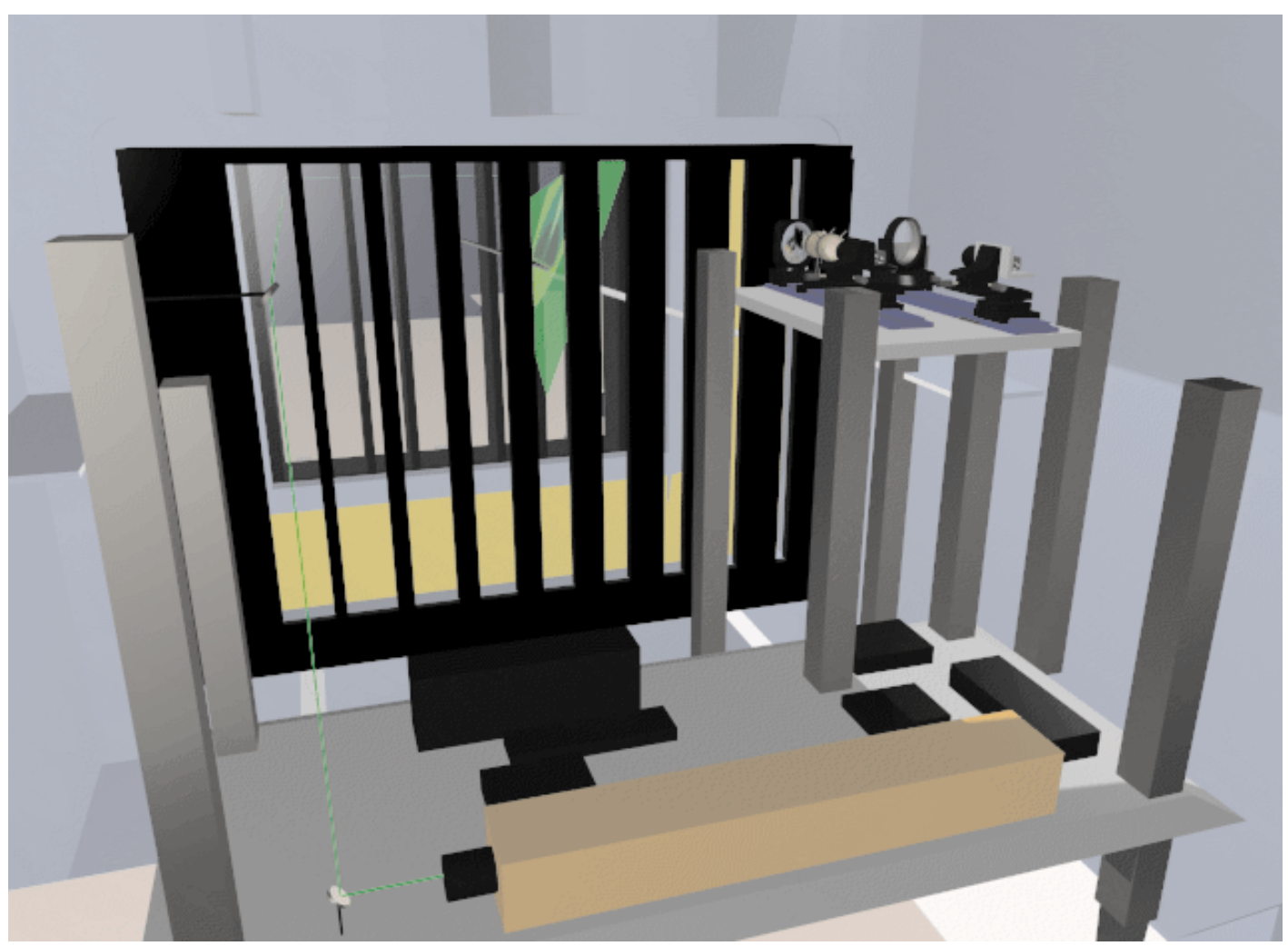

Figure 6.-Illustration of the DGV optics system installed in the UPWT. 


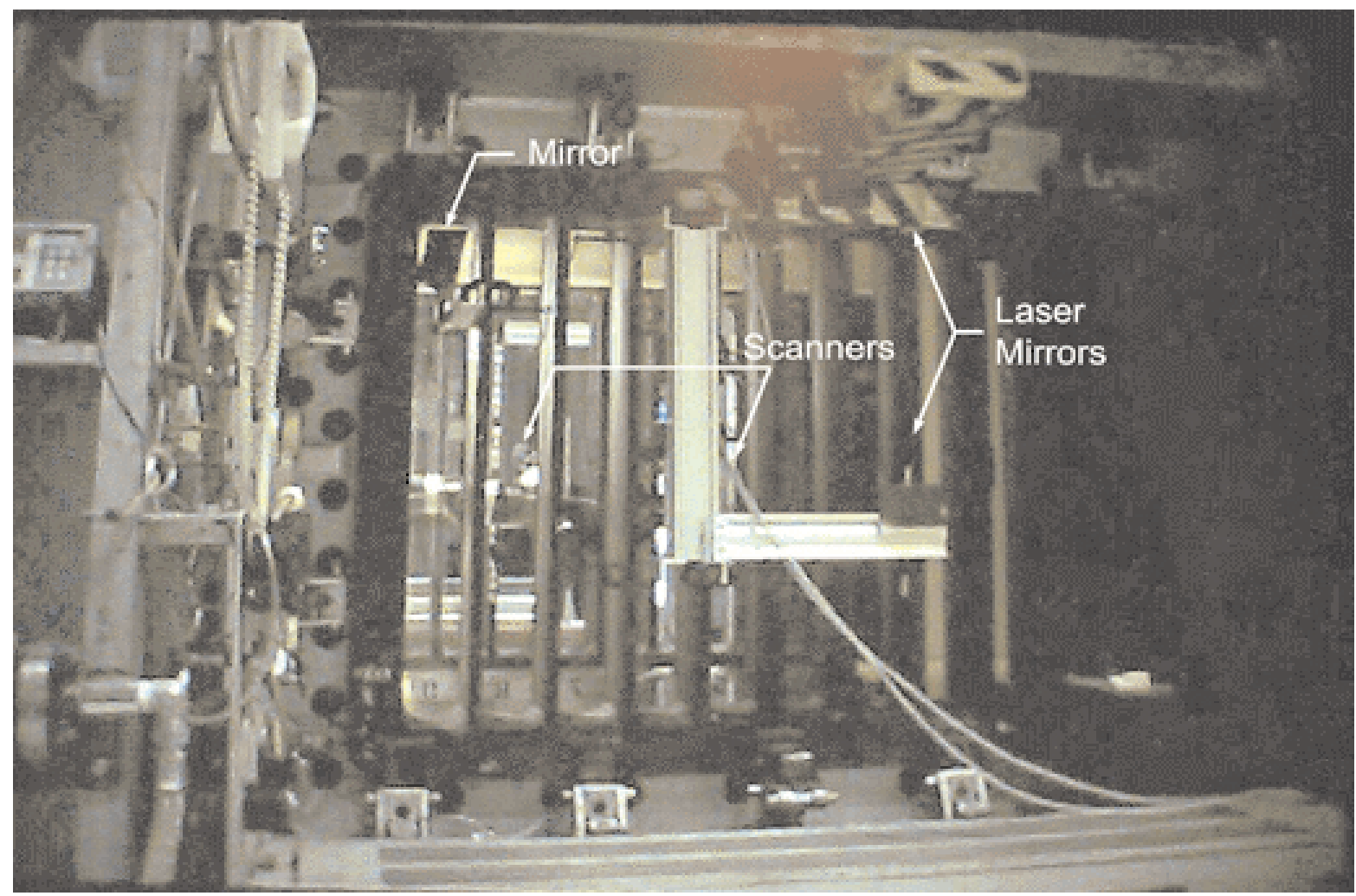

Figure 7.-Photograph of the optical components on the far side of the test section.

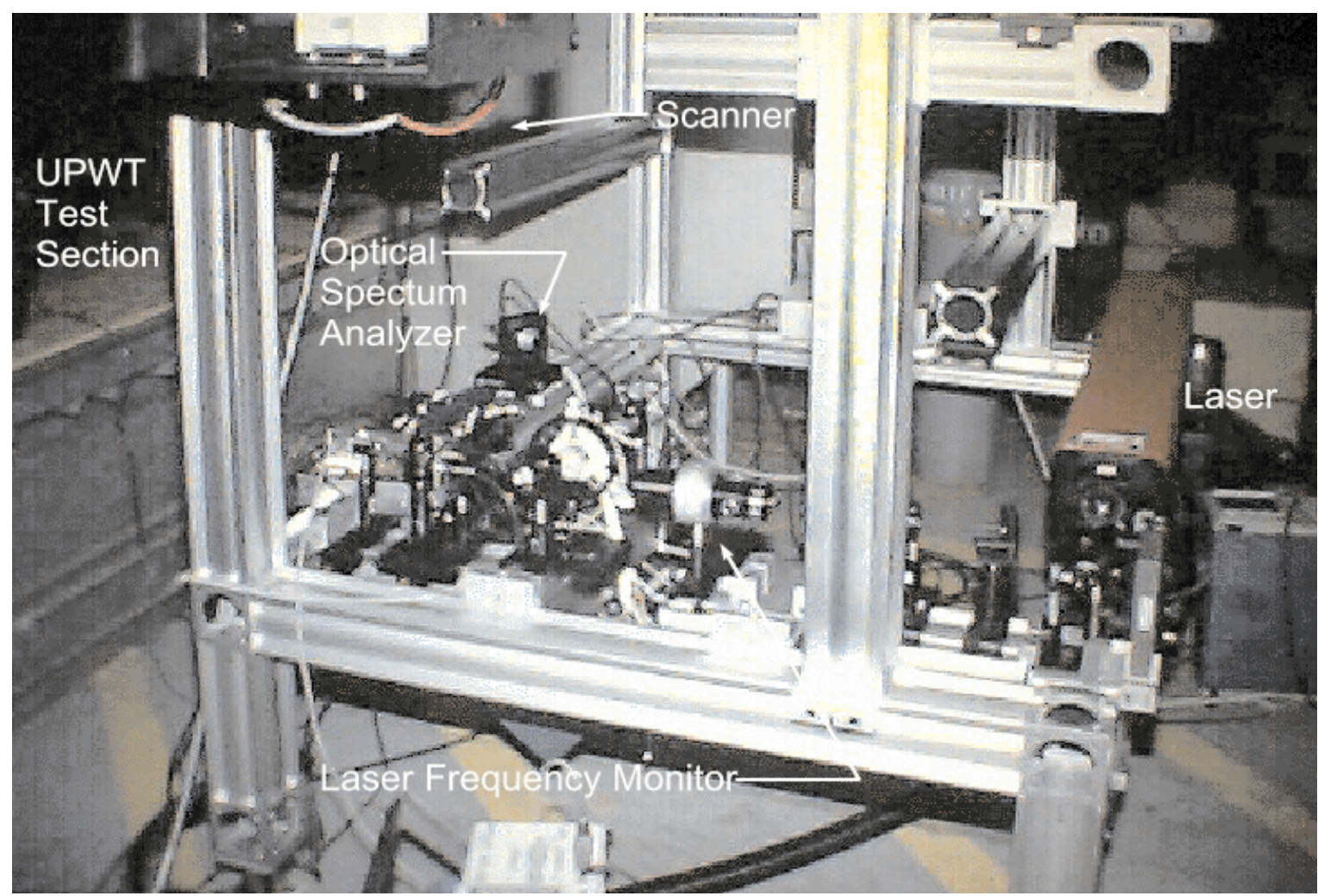

Figure 8.-Photograph of the DGV laser and optical frequency monitoring system. 


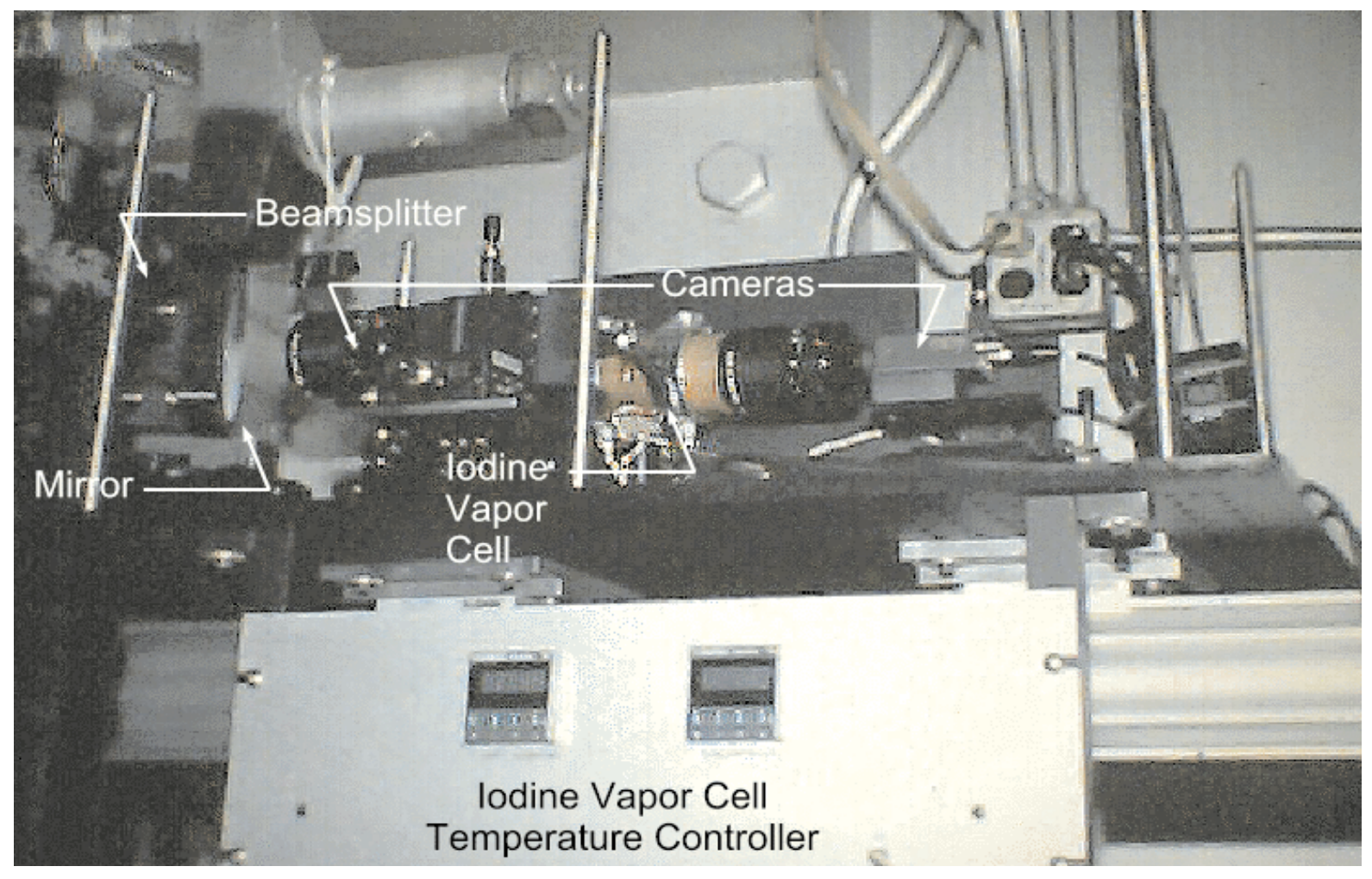

Figure 9.-Photograph of the DGV receiver optical system.

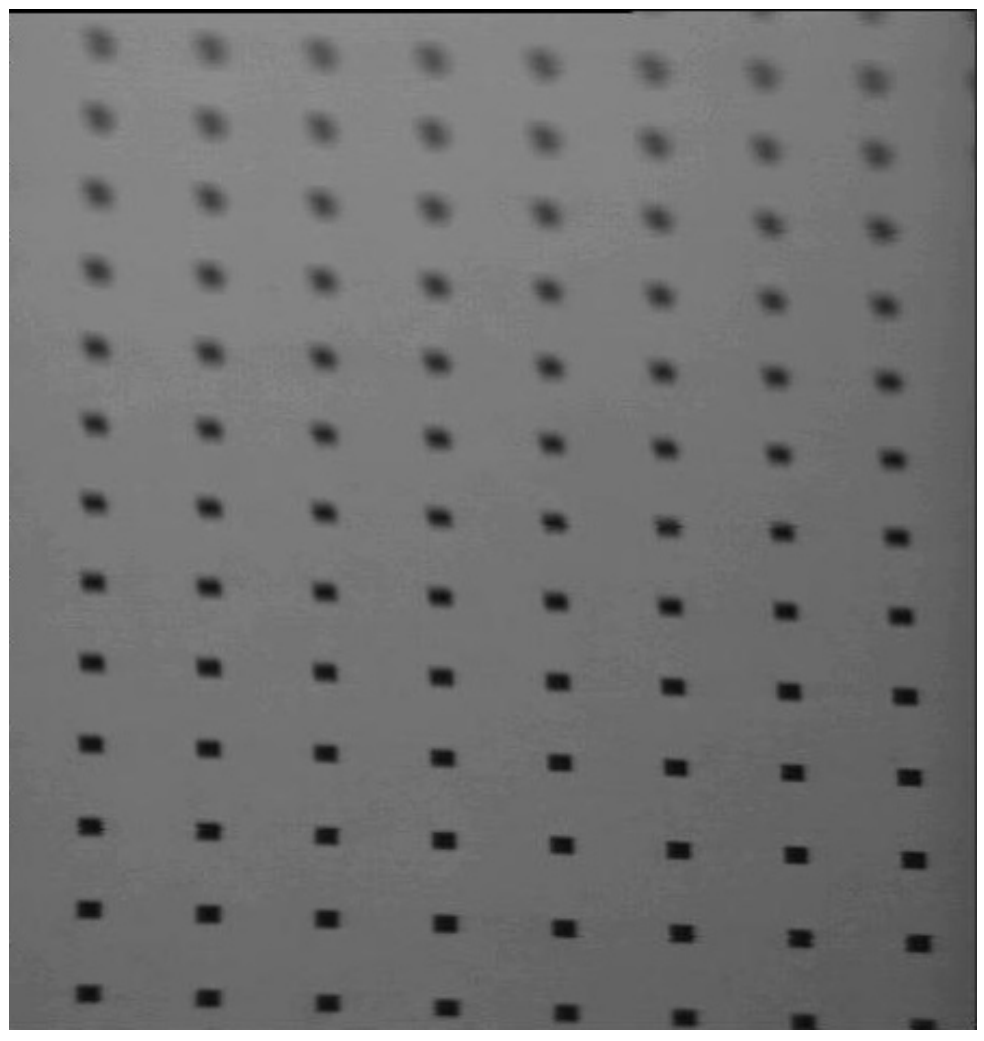

Figure 10.-Image of the alignment target set for Phase I and II. 


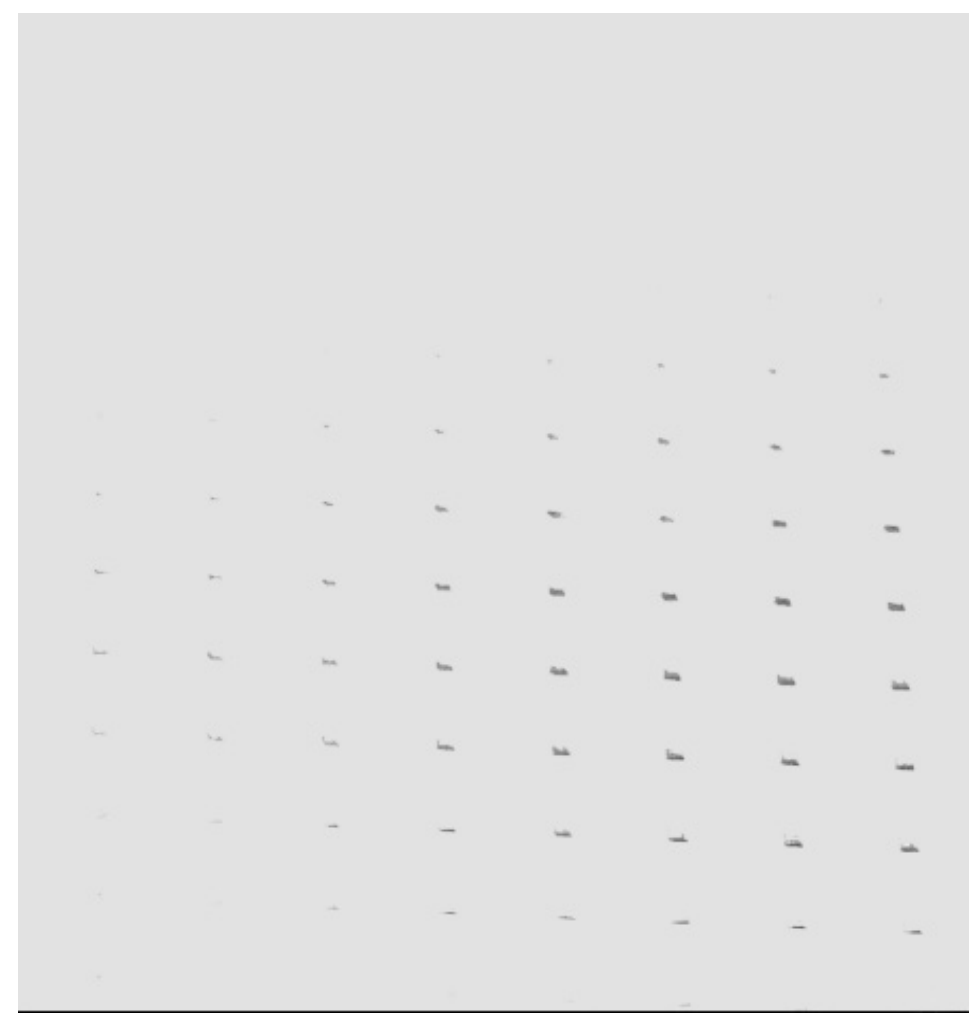

Figure 11.-Normalized image of the alignment target set for Phases I and II.

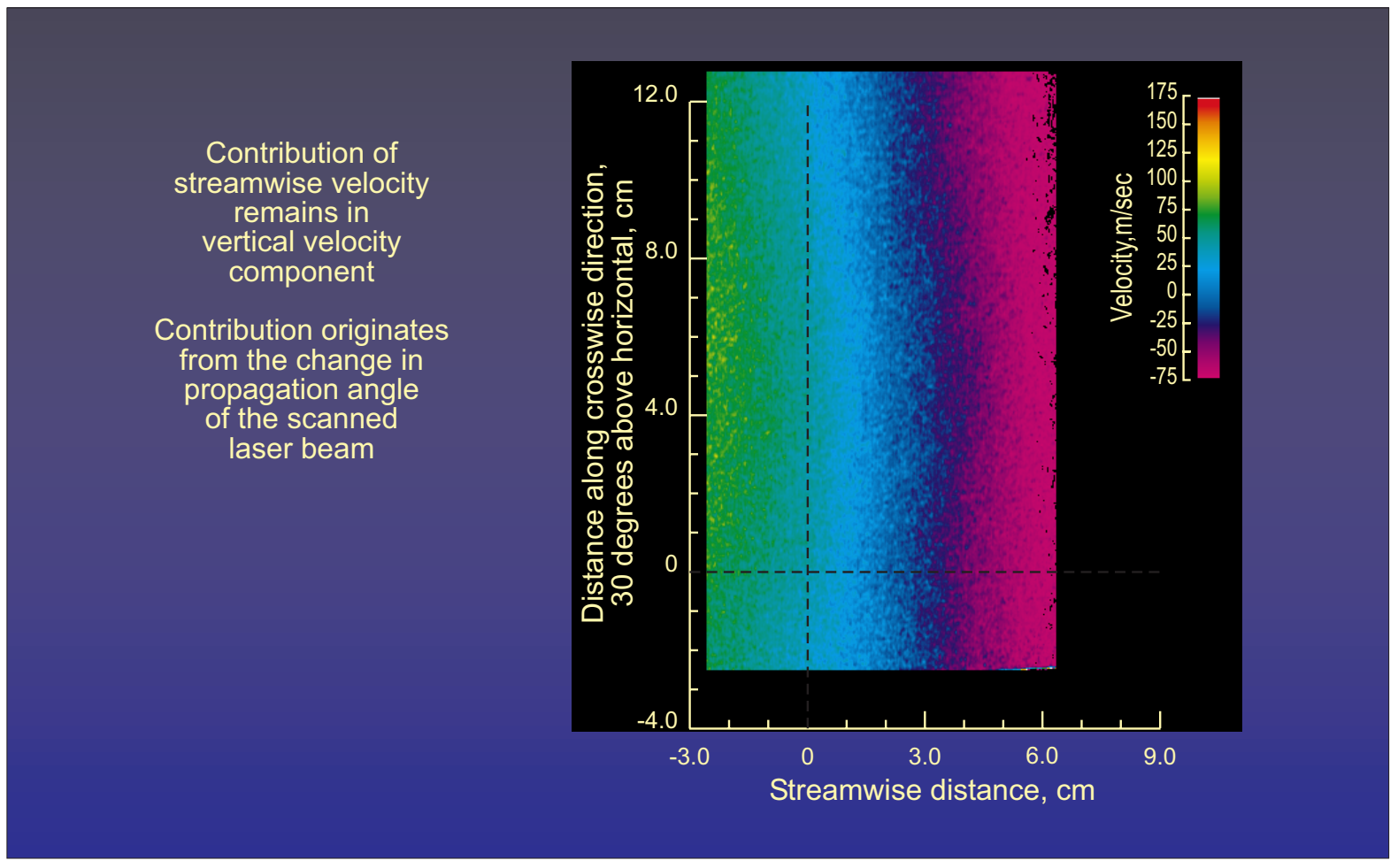

Figure 12.-Map of the vertical velocity component measured by DGV of the empty tunnel flow at Mach 2.5. Streamwise velocity contribution remains in data. 
Contribution of streamwise velocity remains in vertical velocity component

Contribution originates from the change in propagation angle of the scanned laser beam

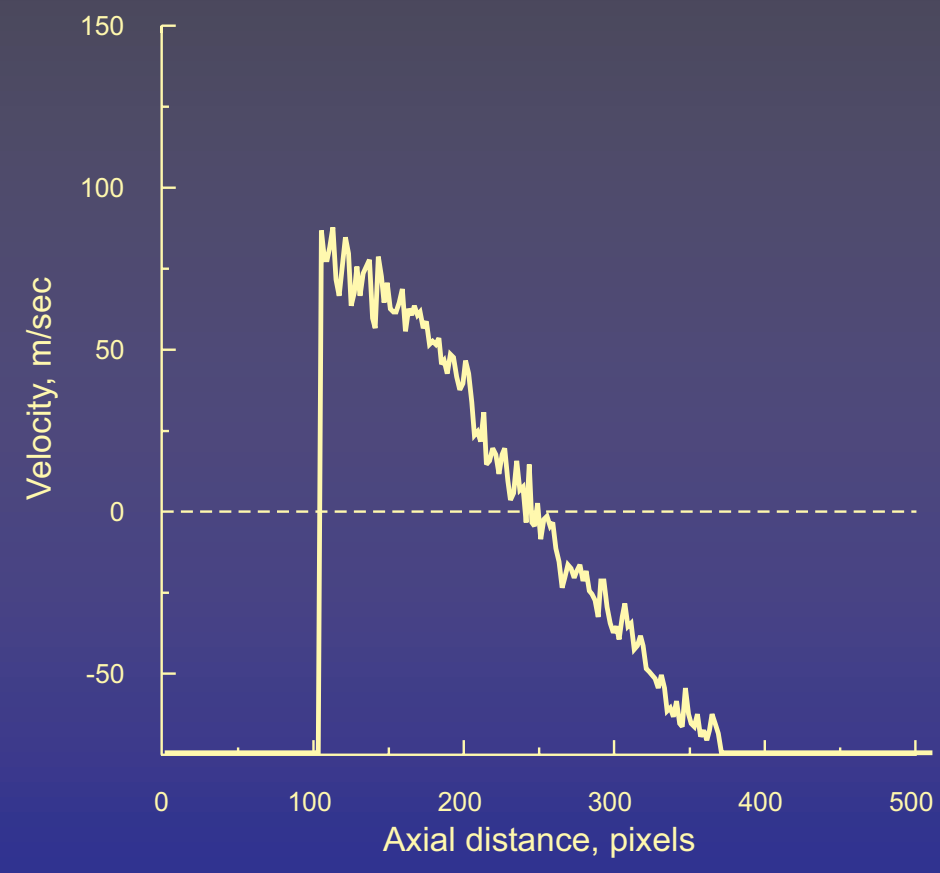

Figure 13.-Streamwise profile of the vertical velocitycomponent measured by DGV of the empty tunnel flow at Mach 2.5. Streamwise velocity contribution remains in data.

Contribution of streamwise velocity removed from

vertical velocity component

Contribution originates from the change in propagation angle of the scanned laser beam

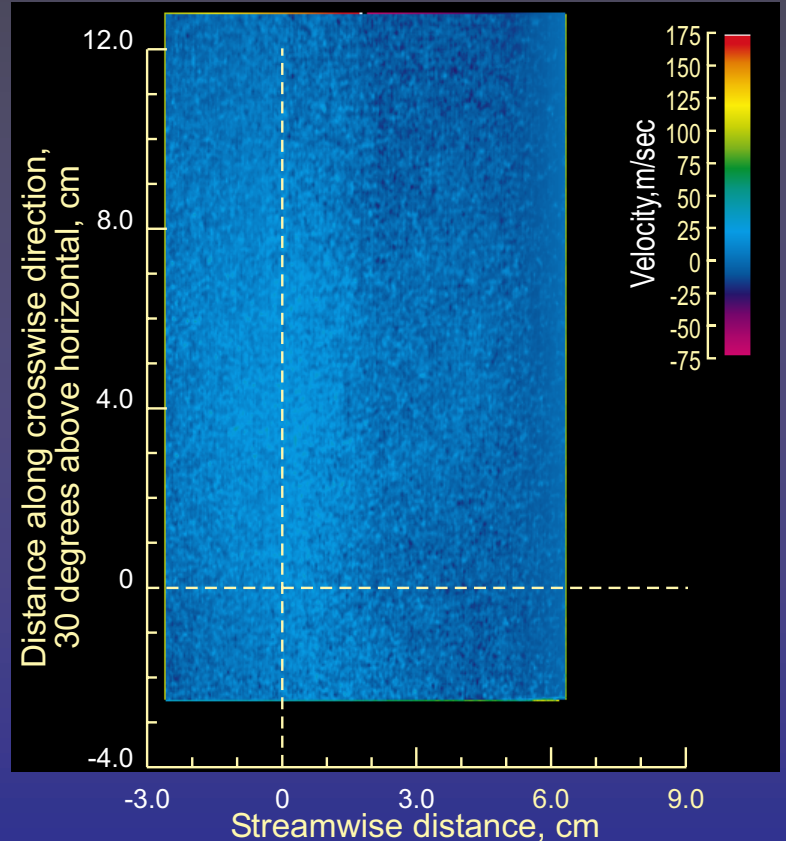

Streamwise distance, $\mathrm{cm}$

Figure 14.-Map of the vertical velocity component measured by DGV of the empty tunnel flow at Mach 2.5. Streamwise velocity contribution removed. 
Contribution of streamwise velocity removed from

vertical velocity component

Contribution originates from the change in propagation angle of the scanned laser beam

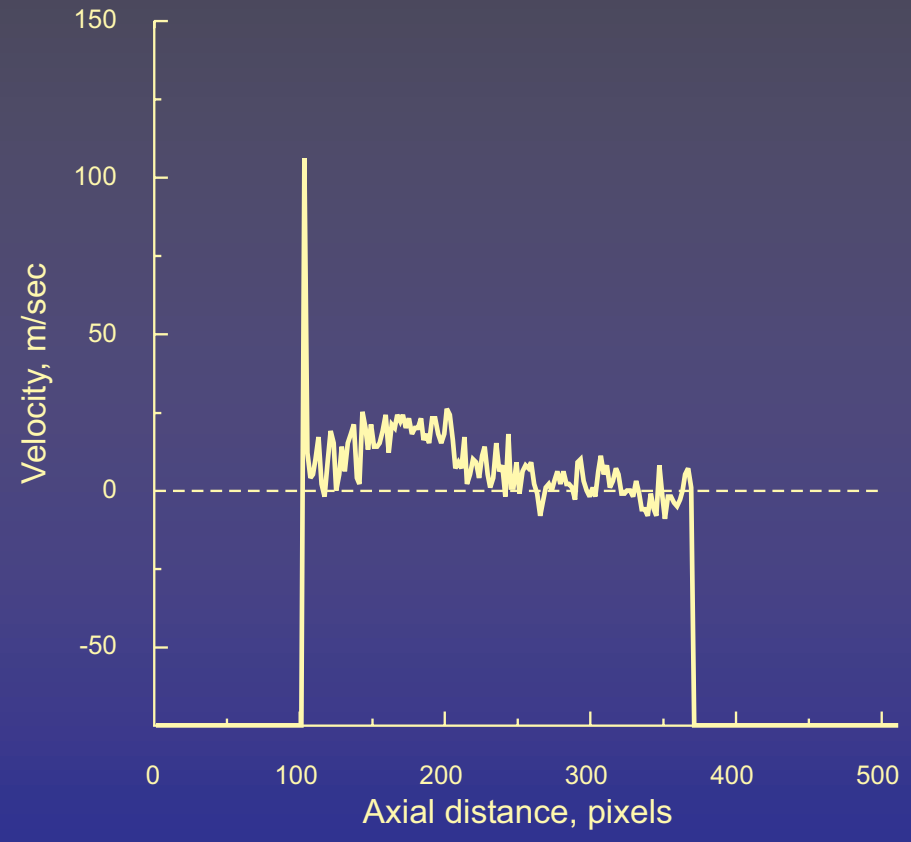

Figure 15.-Streamwise profile of the vertical velocity component measured by DGV of the empty tunnel flow at Mach 2.5. Streamwise velocity contribution removed.

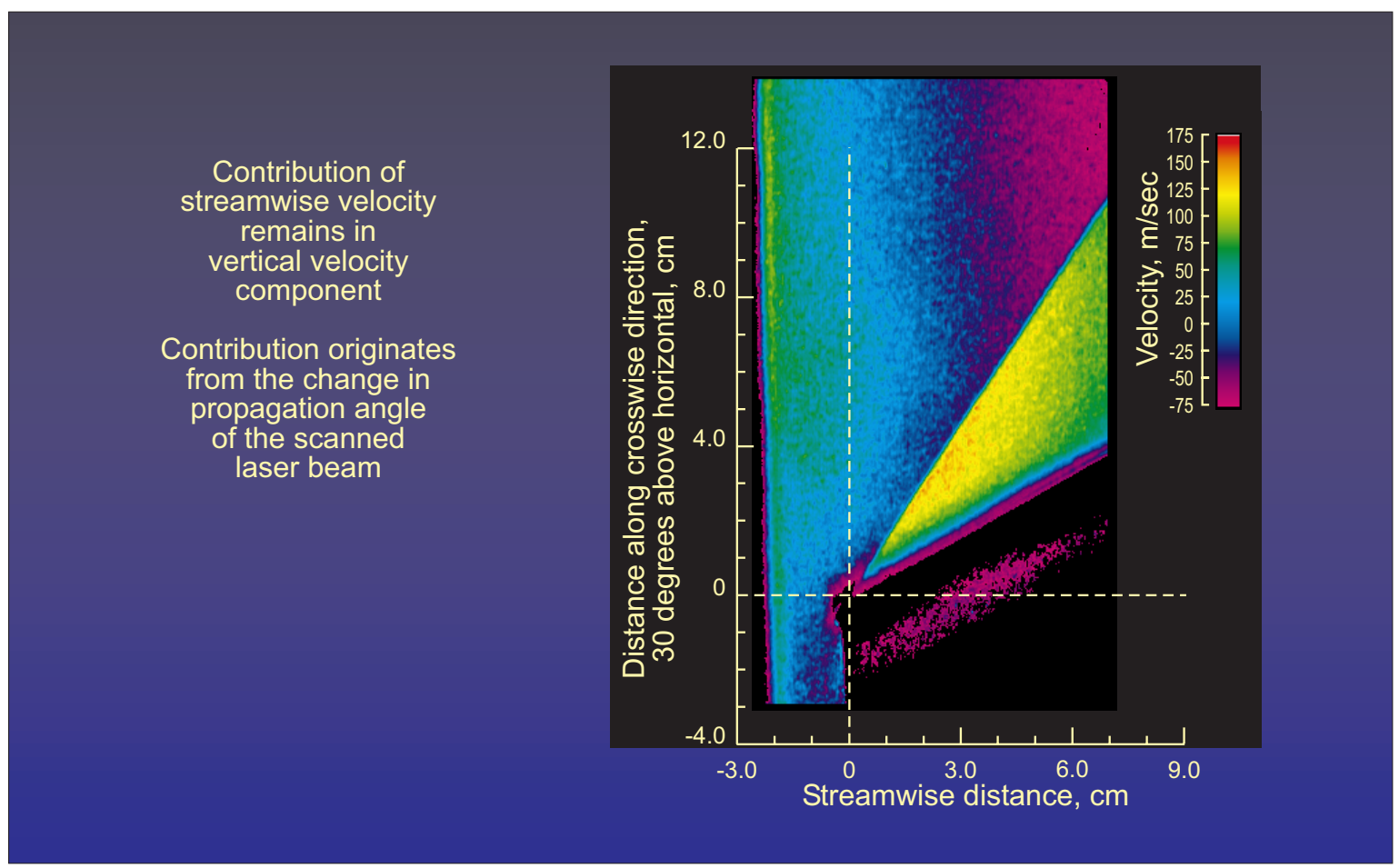

Figure 16.-Map of the vertical velocity component measured by the DGV of the flow above a flat plate inclined to -15 degrees at Mach 2.5. Streamwise velocity contribution remains in data. 


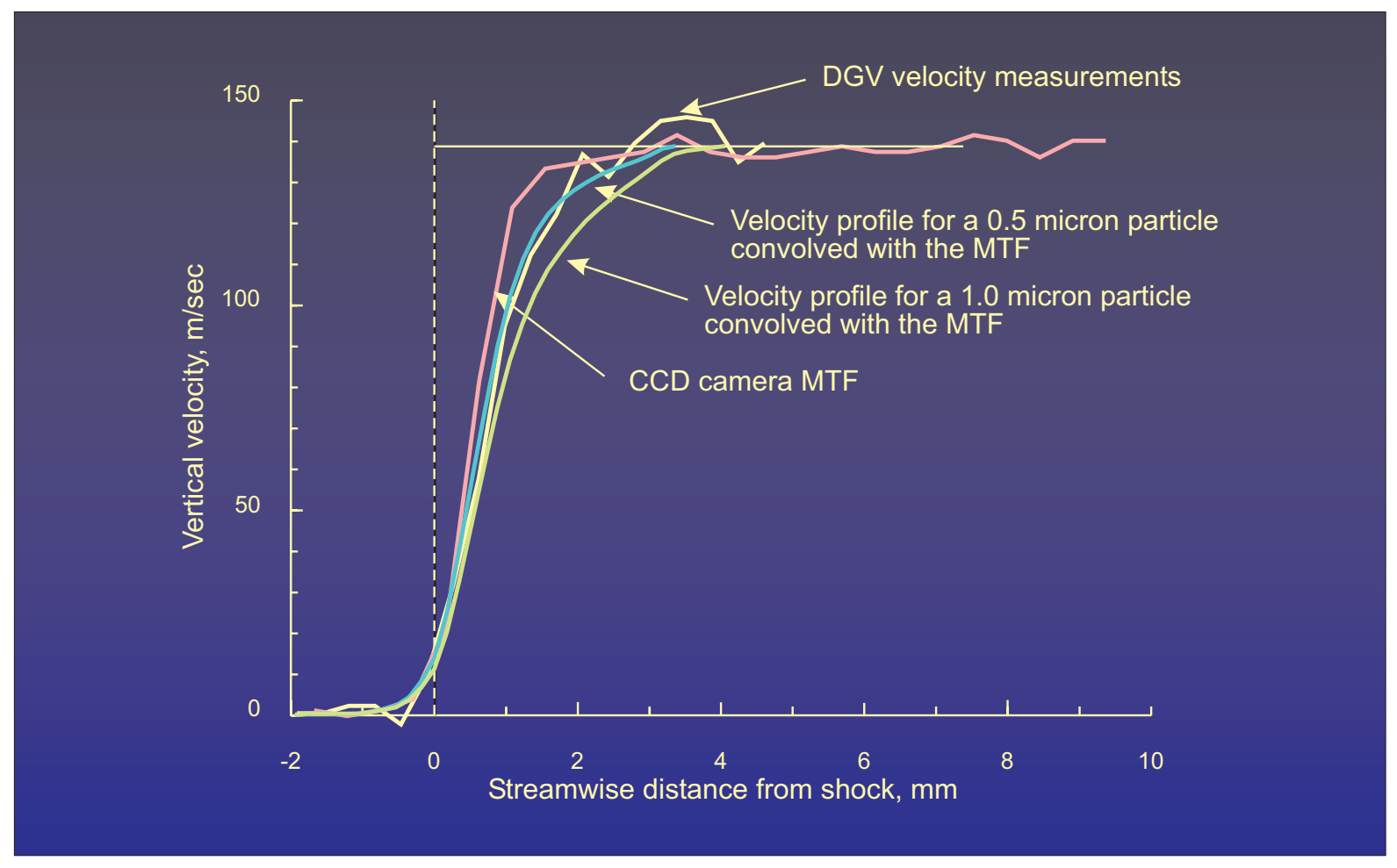

Figure 17.-Comparison of the DGV measurements across the shock with theoretical responses for 0.5 micron and 1.0 micron particles. The CCD camera modulation transfer function indicates the limits of the instrument

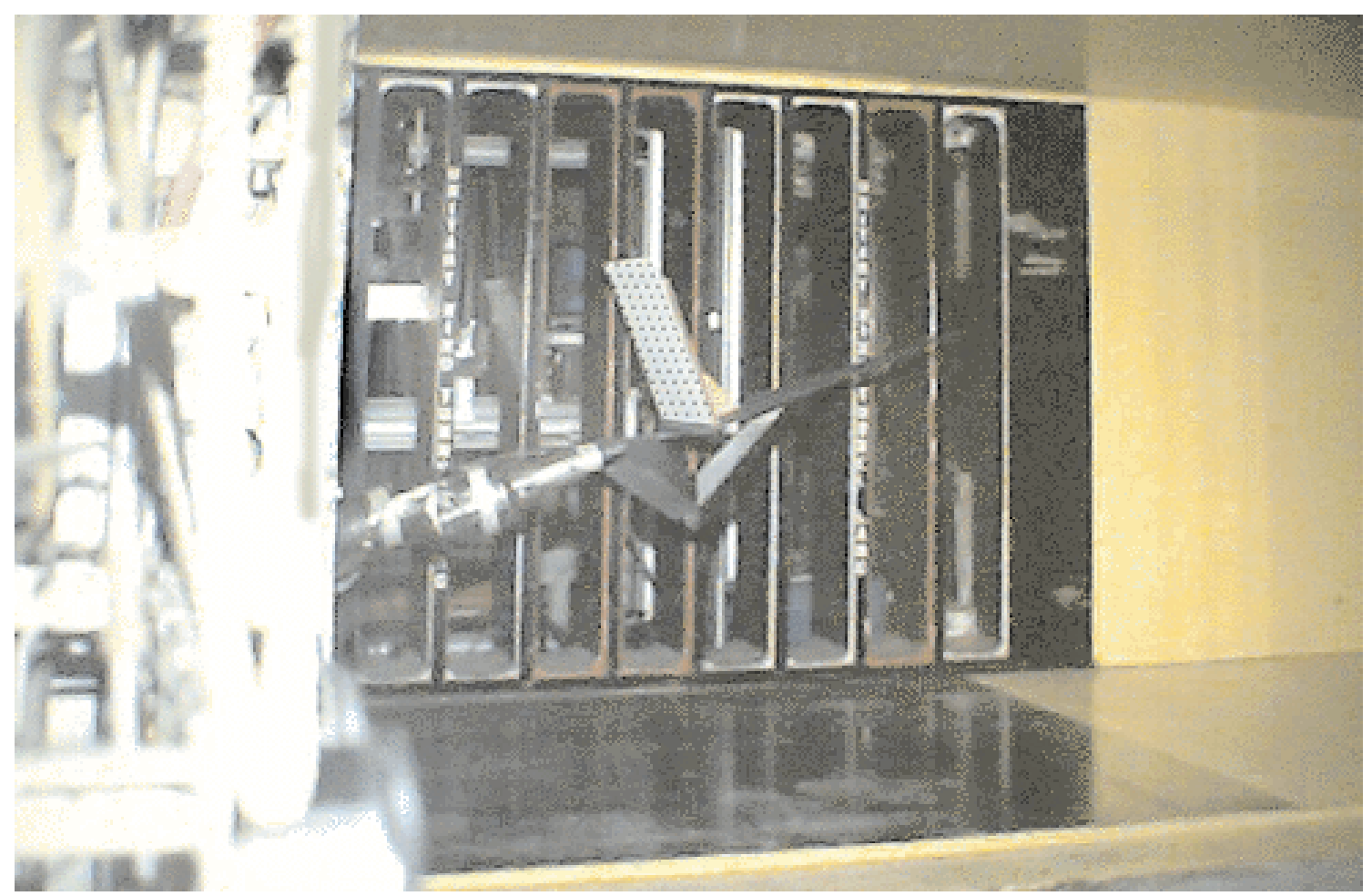

Figure 18.-Photograph of the alignment target placed at the 95-percent chord location on the 75 -degree delta wing. 


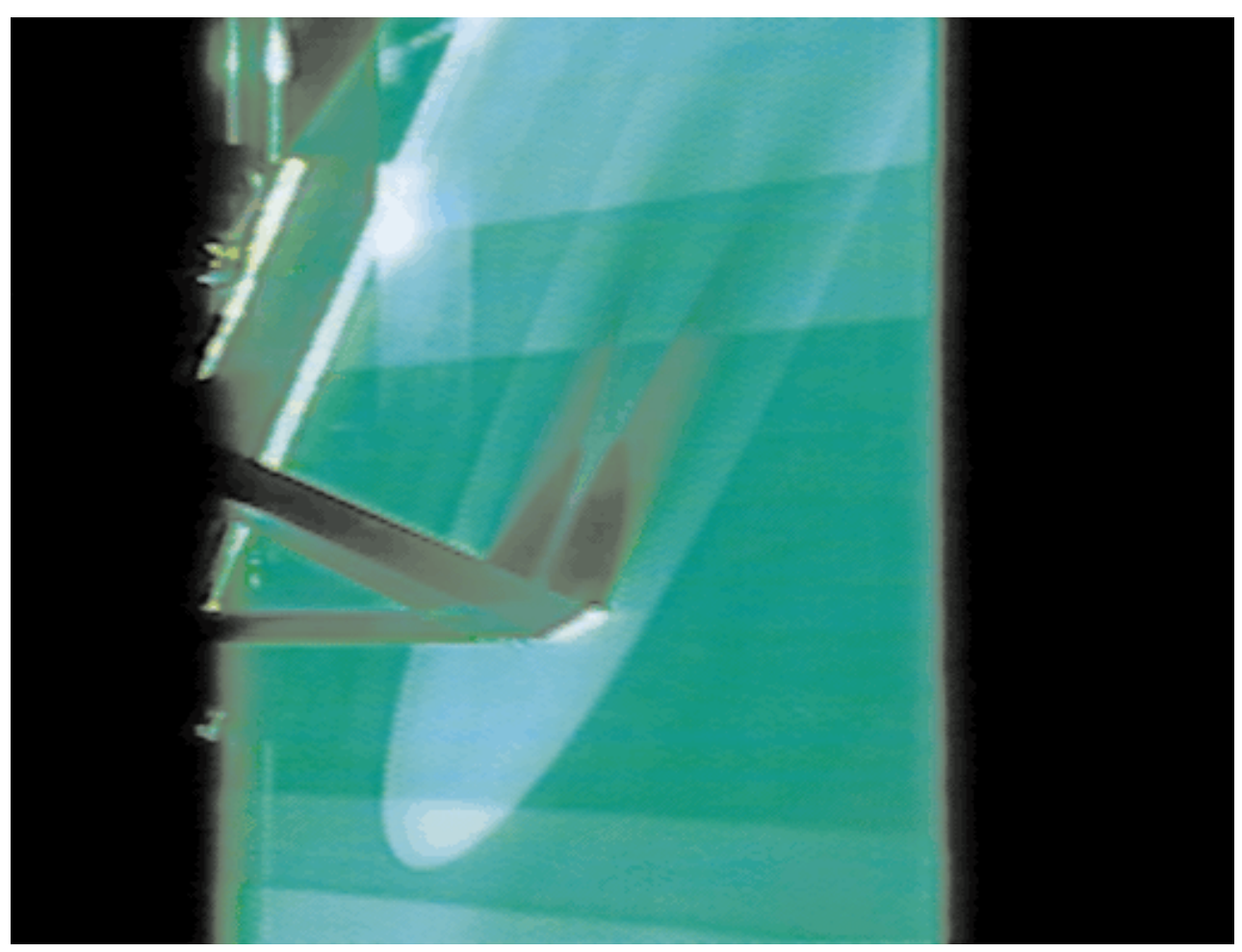

Figure 19.-Photograph of the laser light sheet above a 75-degree delta wing at the 95-percent chord location at Mach 2.8.

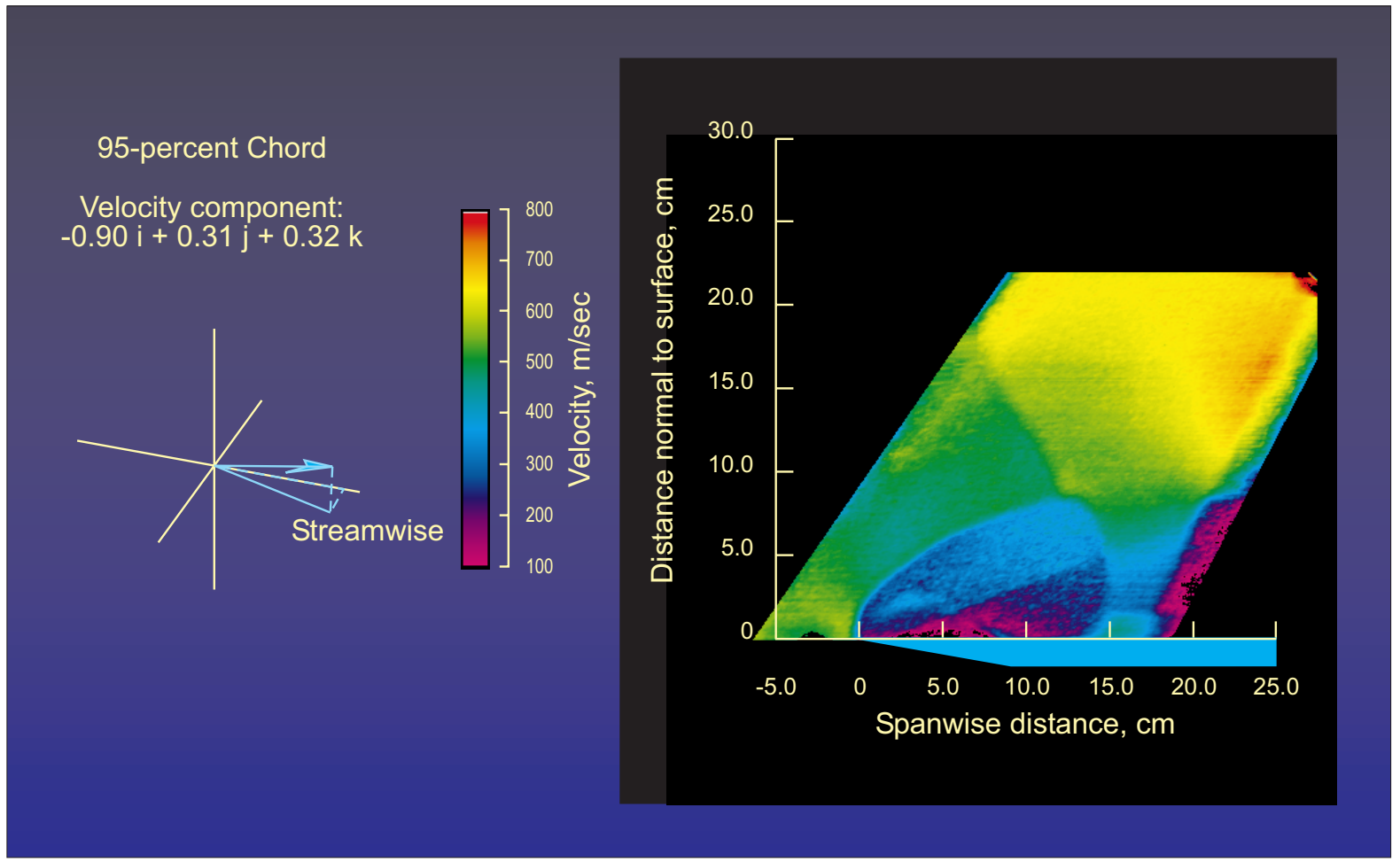

Figure 20.-Map of the velocity component: $-0.90 \mathrm{i}+0.31 \mathrm{j}+0.32 \mathrm{k}$ measured by the DGV of the vortical flow above a 75-degree delta wing at the 95 -percent chord location at Mach 2.8. 


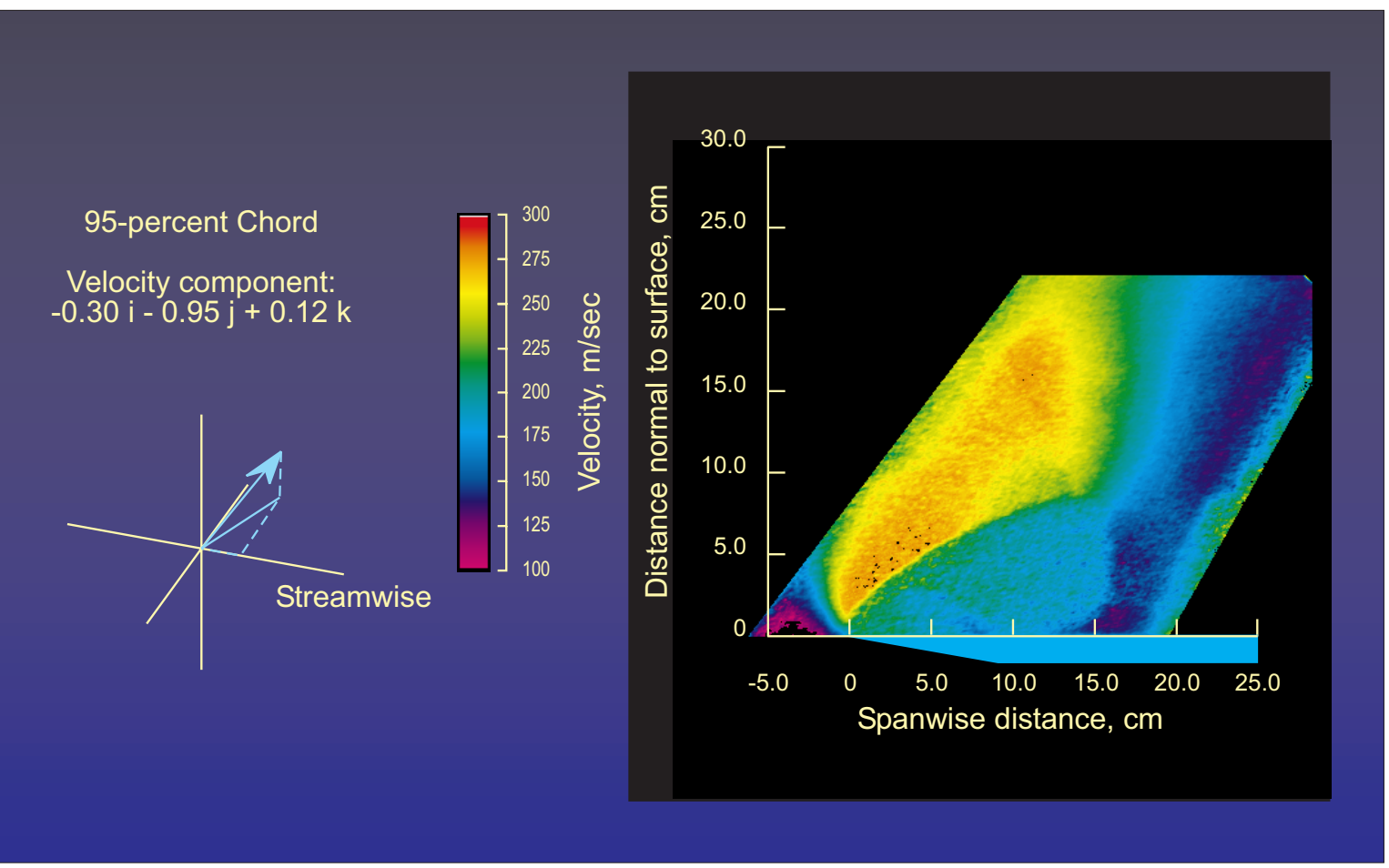

Figure 21.-Map of the velocity component: $-0.30 \mathrm{i}-0.95 \mathrm{j}+0.12 \mathrm{k}$ measured by the DGV of the vortical flow above a 75 -degree delta wing at the 95 -percent chord location at Mach 2.8.

95-percent Chord

Velocity component: $-0.57 i+0.40 j+0.72 k$

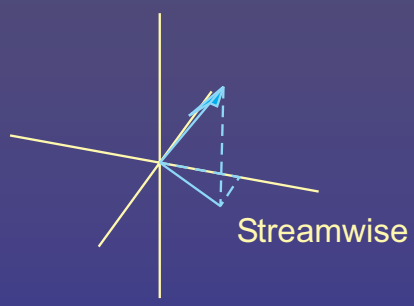

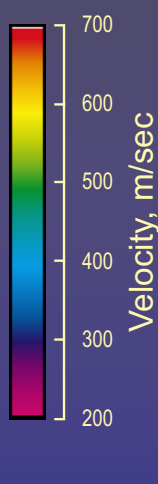

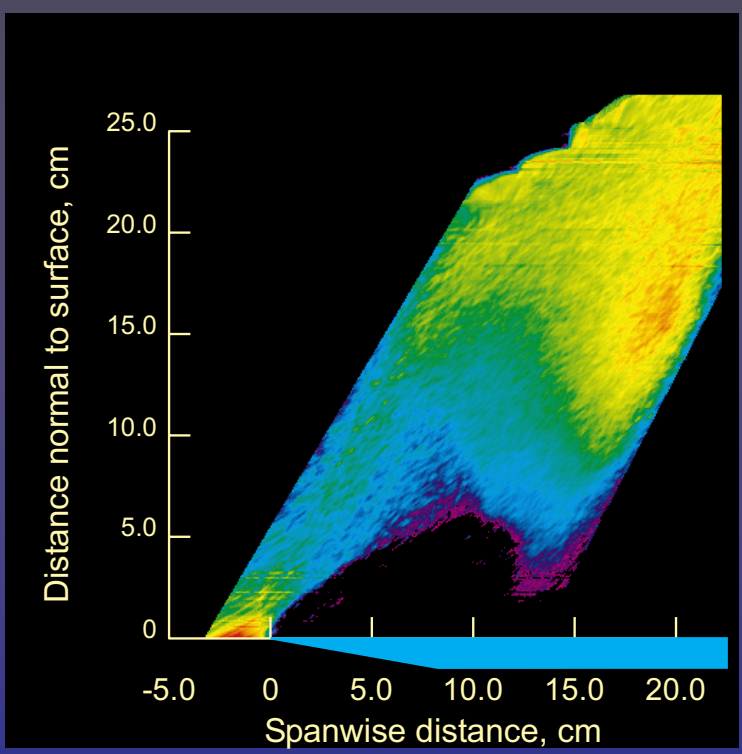

Figure 22.-Map of the velocity component: $-0.57 \mathrm{i}+0.40 \mathrm{j}+0.72 \mathrm{k}$ measured by the DGV of the vortical flow above a 75 -degree delta wing at the 95 -percent chord location at Mach 2.8. 


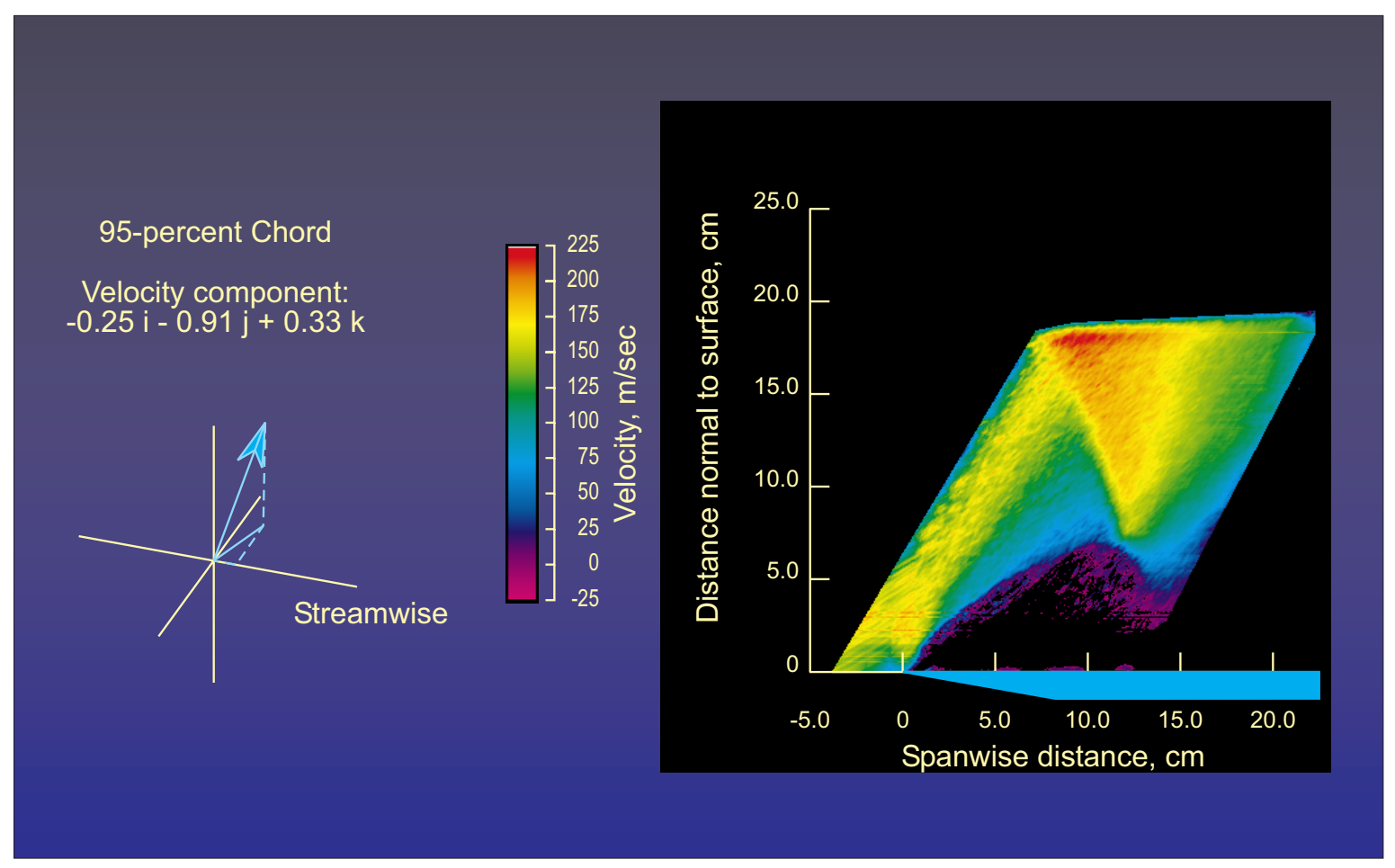

Figure 23.-Map of the velocity component: $-0.25 \mathrm{i}-0.91 \mathrm{j}+0.33 \mathrm{k}$ measured by the DGV of the vortical flow above a 75 -degree delta wing at the 95 -percent chord location at Mach 2.8. 